\title{
Blow up and Decay for a Class of $p$-Laplacian Hyperbolic Equation with Logarithmic Nonlinearity
}

\author{
Ying $\mathrm{Chu}^{*}$, Yuqi Wu and Libo Cheng
}

\begin{abstract}
In this paper, we study an initial boundary value problem for a $p$-Laplacian hyperbolic equation with logarithmic nonlinearity. By combining the modified potential well method with the Galerkin method, the existence of the global weak solution is studied, and the polynomial and exponential decay estimation under certain conditions are also given. Moreover, by using the concavity method and other techniques, we obtain the blow up results at finite time.
\end{abstract}

\section{Introduction}

In this paper, we study the following problem

$$
\begin{cases}u_{t t}-\Delta_{p} u-\Delta u_{t}=|u|^{q-2} u \log |u|, & (x, t) \in \Omega \times(0, T), \\ u(x, t)=0, & (x, t) \in \partial \Omega \times(0, T), \\ u(x, 0)=u_{0}(x), \quad u_{t}(x, 0)=u_{1}(x), & x \in \Omega,\end{cases}
$$

where $\Omega \subset R^{n}(n \geq 1)$ is a bound domain with smooth boundary $\partial \Omega, p, q$ are constants and they satisfy $2<p<q<p(1+2 / n)$.

During the past decades, many papers have been devoted to the study of qualitative properties of solutions for hyperbolic equation, with many remarkable results. We can refer to $1,4,5,9,12,14,16,18,21,24,25]$ and the references therein.

The semilinear hyperbolic equation

$$
u_{t t}-\Delta u=f(u)
$$

was introduced by D'Alembert [1] to model the propagation of waves along vibrating elastic string. Sattinger 21] first established a method of potential wells to deal with the global existence of solutions to the problem $(1.2)$. Payne and Sattinger $[18$ showed that the solution blows up in finite time. In [9], the model $[1.2$ was studied by Lian et

Received October 11, 2021; Accepted January 24, 2022.

Communicated by François Hamel.

2020 Mathematics Subject Classification. 35A01, 35L20, 35B40, 35B44.

Key words and phrases. $p$-Laplacian hyperbolic equation, global existence, energy decay estimates, blow up, logarithmic nonlinearity.

*Corresponding author. 
al., where $f(u)=u \ln |u|^{k}$, they modified the potential well method and combined with logarithmic Sobolev inequality to obtain the global existence of the solution and the blow up result under the condition of different initial energy. Later, in the case of nonlinearity $f(u)=|u|^{p} \ln u$, the problem $[1.2)$ is also considered by Lian et al. [10], and they establish the global existence and finite time blow up of solutions.

For the classical strongly damped hyperbolic equation

$$
u_{t t}-\Delta u-\Delta u_{t}=f(u)
$$

Webb [24] gave the existence, uniqueness and the asymptotic behavior of the solution. In the case of nonlinearity $f(u)=u \log |u|^{2}$, the model (1.3) was studied by Ma and Fang [14], they proved the existence of global weak solutions, derived the energy decay estimates and blow up at infinity.

In [26], $\mathrm{Xu}$ and $\mathrm{Su}$ studied the parabolic equation with strongly damped term

$$
u_{t}-\Delta u_{t}-\Delta u=u^{p}
$$

where $p>1$, they proved global existence, asymptotic behavior and finite time blow up of solution with initial energy $J\left(u_{0}\right) \leq d$. Moreover, they also obtained finite time blow up with high initial energy $J\left(u_{0}\right)>d$ by comparison principle. The relevant equations have also been studied in 11,23.

For the initial boundary value problem of strongly damped hyperbolic equation

$$
u_{t t}-\Delta u-\omega \Delta u_{t}+\mu u_{t}=f(u),
$$

where $\omega \geq 0, \mu>-\omega \lambda_{1}, \lambda_{1}$ is the first eigenvalue of the operator $-\Delta$ under homogeneous Dirichlet boundary conditions. In the case of nonlinearity $f(u)=|u|^{p-2} u$, Gazzolaa and Squassina [4] investigated the problem (1.4), obtained the existence of global weak solutions by the potential well method, and also proved the blow up results at finite time. In the case of nonlinearity $f(u)=u \ln |u|$, Lian and Xu $[9]$ proved the local existence of the weak solution by the contraction mapping principle, and under the framework of potential well, studied the energy decay and global existence, and the blow up result of the solution at infinite time.

In recent years, the $p$-Laplacian parabolic equation with logarithmic nonlinearity is studied extensively. Le et al. [7] studied the following $p$-Laplacian parabolic equation with logarithmic source term

$$
u_{t}-\Delta_{p} u=|u|^{p-2} u \log |u|,
$$

they obtained results of existence or nonexistence of global weak solutions and finite time blow-up. Moreover, the large time decay of global weak solutions was studied. 
For parabolic equations with strongly damped and logarithmic source terms

$$
u_{t}-\Delta u_{t}-\Delta_{p} u=|u|^{q-2} u \log |u| \text {. }
$$

In the case where $p$ is equal to $q$, Nhan and Truong [15] obtained results of global existence and finite time blow up of the weak solution by using the potential well method and logarithmic Sobolev inequality (see $[19,20]$ ) to the problem 1.5 . When $2<p<q<$ $p(1+2 / n)$, He et al. [6] investigated the decay and finite time blow up of the weak solutions. When $1<p \leq q<p^{*}$, the problem (1.5) was studied by Ding and Zhou in [2], they mainly discuss the properties of global existence, blow up at infinity, and finite time blow up.

As shown in the previous works, the research on the properties of solutions of $p$ Laplacian parabolic equations is relatively perfect. There are not much literature for strongly damped $p$-Laplace hyperbolic equations with logarithmic source term. For the problem (1.1), the appearance of $p$-Laplacian operator $\Delta_{p} u$ and logarithmic source terms $|u|^{q-2} u \log |u|$ bring some difficulties, so that we cannot use Sobolev inequality as in reference [14. Here, we modify the potential well method and combine with the concavity method to obtain the global existence of the weak solution, energy decay estimate, and the blow up result of the solution at a finite time.

The paper is organized as follows. In Section 2, some useful lemmas and main results are included. Section 3 is devoted to discussing global existence and energy decay estimate for the problem (1.1). In the last section, we prove the blow up theorem and give the lower and upper bounds for blow up time.

\section{Preliminaries and main results}

To get the main results of this paper, we first introduce some notations, definitions, and lemmas. For convenience, we let

$$
X_{0}=\left\{\left(u, u_{t}\right) \mid u \in W_{0}^{1, p}(\Omega) \backslash\{0\}, u_{t} \in L^{2}(\Omega) \backslash\{0\}\right\} .
$$

Throughout this paper, we denote the norm of $L^{s}(\Omega)$ for $1 \leq s \leq \infty$ by $\|\cdot\|_{s}$ and the norm of $W_{0}^{1, p}(\Omega)$ by $\|\cdot\|_{1, p}$. That is, for any $u \in L^{s}(\Omega)$,

$$
\|u\|_{s}=\|u\|_{L^{s}(\Omega)}= \begin{cases}\left(\int_{\Omega}|u(x)|^{s} d x\right)^{1 / s} & \text { if } 1 \leq s<\infty \\ {\operatorname{ess} \sup _{x \in \Omega}|u(x)|} \text { if } s=\infty\end{cases}
$$

and for any $u \in W_{0}^{1, p}(\Omega)$,

$$
\|u\|_{1, p}=\|u\|_{W_{0}^{1, p}(\Omega)}=\left(\|u\|_{p}^{p}+\|\nabla u\|_{p}^{p}\right)^{1 / p} .
$$


We denote the dual space of $W_{0}^{1, p}(\Omega)$ by $W_{0}^{-1, p^{\prime}}(\Omega)$, where $p^{\prime}=p /(p-1)$. For $\left(u, u_{t}\right) \in X_{0}$, let us introduce some functionals and sets as follows:

$$
\begin{gathered}
E(t)=\frac{1}{2}\left\|u_{t}\right\|_{2}^{2}+\frac{1}{p}\|\nabla u\|_{p}^{p}-\frac{1}{q} \int_{\Omega}|u|^{q} \log |u| d x+\frac{1}{q^{2}}\|u\|_{q}^{q}, \\
J(u)=\frac{1}{p}\|\nabla u\|_{p}^{p}-\frac{1}{q} \int_{\Omega}|u|^{q} \log |u| d x+\frac{1}{q^{2}}\|u\|_{q}^{q}, \\
I(u)=\|\nabla u\|_{p}^{p}-\int_{\Omega}|u|^{q} \log |u| d x \\
\mathcal{N}=\left\{u \in W_{0}^{1, p}(\Omega) \backslash\{0\} \mid I(u)=0\right\} \\
d=\inf _{u \in X_{0}}\left\{\sup _{\lambda>0} J(\lambda u)\right\}=\inf _{u \in \mathcal{N}} J(u),
\end{gathered}
$$

$W_{1}=\left\{\left(u, u_{t}\right) \in X_{0} \mid E(t)<d\right\}, \quad W_{2}=\left\{\left(u, u_{t}\right) \in X_{0} \mid E(t)=d\right\}, \quad W=W_{1} \cup W_{2}$,

$W_{1}^{+}=\left\{\left(u, u_{t}\right) \in W_{1} \mid I(u) \geq 0\right\}, \quad W_{2}^{+}=\left\{\left(u, u_{t}\right) \in W_{2} \mid I(u) \geq 0\right\}, \quad W^{+}=W_{1}^{+} \cup W_{2}^{+}$, $W_{1}^{-}=\left\{\left(u, u_{t}\right) \in W_{1} \mid I(u)<0\right\}, \quad W_{2}^{+}=\left\{\left(u, u_{t}\right) \in W_{2} \mid I(u)<0\right\}, \quad W^{-}=W_{1}^{-} \cup W_{2}^{-}$.

By the definition of $J(u)$ and $I(u)$, we get that

$$
J(u)=\frac{1}{q} I(u)+\left(\frac{1}{p}-\frac{1}{q}\right)\|\nabla u\|_{p}^{p}+\frac{1}{q^{2}}\|u\|_{q}^{q} .
$$

We give the definition of the weak solution to the problem $(1.1)$ as follows:

Definition 2.1. We define a function $u \in L^{\infty}\left(0, T ; W_{0}^{1, p}(\Omega)\right)$ with $u_{t} \in L^{2}\left(0, T ; H_{0}^{1}(\Omega)\right)$ to be a weak solution of problem (1.1) over $[0, T]$, if it satisfies the initial condition $u(x, 0)=$ $u_{0} \in W_{0}^{1, p}(\Omega) \backslash\{0\}, u_{t}(x, 0)=u_{1} \in L^{2}(\Omega) \backslash\{0\}$, and

$$
\begin{aligned}
& \left(u_{t}, w\right)+\int_{0}^{t}\left(|\nabla u|^{p-2} \nabla u, \nabla w\right) d \tau+(\nabla u, \nabla w) \\
= & \left(u_{1}, w\right)+\left(\nabla u_{0}, \nabla w\right)+\int_{0}^{t}\left(|u|^{q-2} u \log |u|, w\right) d \tau
\end{aligned}
$$

for any $w \in W_{0}^{1, p}(\Omega)$, and for a.e. $t \in[0, T]$.

For a fixed $u \in W_{0}^{1, p}(\Omega) \backslash\{0\}$, we consider the function $j(\lambda)=J(\lambda u)$ for $\lambda>0$. This map was introduced by Drabek and Pohozaev [3]. We list some properties of $j$ in the following lemma.

Lemma 2.2. Let $u \in W_{0}^{1, p}(\Omega) \backslash\{0\}$, then the following results hold:

(1) $\lim _{\lambda \rightarrow 0^{+}} j(\lambda)=0, \lim _{\lambda \rightarrow+\infty} j(\lambda)=-\infty$;

(2) There exists a unique $\lambda_{*}>0$ such that $j^{\prime}\left(\lambda_{*}\right)=0$;

(3) $j(\lambda)$ is increasing on $\lambda \in\left(0, \lambda_{*}\right)$, decreasing on $\lambda \in\left(\lambda_{*},+\infty\right)$; 
(4) $I(\lambda u)\left\{\begin{array}{ll}>0, & \lambda \in\left(0, \lambda_{*}\right), \\ <0, & \lambda \in\left(\lambda_{*},+\infty\right),\end{array}\right.$ and $I\left(\lambda_{*} u\right)=0$.

Proof. For $u \in W_{0}^{1, p}(\Omega) \backslash\{0\}$, by the definition of $j$, we have

$$
j(\lambda)=\frac{\lambda^{p}}{p}\|\nabla u\|_{p}^{p}-\frac{\lambda^{q}}{q} \int_{\Omega}|u|^{q} \log |u| d x-\frac{\lambda^{q} \log \lambda}{q}\|u\|_{q}^{q}+\frac{\lambda^{q}}{q^{2}}\|u\|_{q}^{q},
$$

it is obvious that (1) holds due to $\|u\|_{q} \neq 0$. Through direct calculation, we get

$$
j^{\prime}(\lambda)=\lambda^{p-1}\left(\|\nabla u\|_{p}^{p}-\lambda^{q-p} \int_{\Omega}|u|^{q} \log |u| d x-\lambda^{q-p} \log \lambda\|u\|_{q}^{q}\right) .
$$

Let $k(\lambda)=\lambda^{1-p} j^{\prime}(\lambda)$, we have

$$
k^{\prime}(\lambda)=-\lambda^{q-p-1}\left((q-p) \int_{\Omega}|u|^{q} \log |u| d x+(q-p) \log \lambda\|u\|_{q}^{q}+\|u\|_{q}^{q}\right) .
$$

Hence, by taking

$$
\lambda_{1}=\exp \left(\frac{(q-p) \int_{\Omega}|u|^{q} \log |u| d x+\|u\|_{q}^{q}}{(p-q)\|u\|_{q}^{q}}\right)>0
$$

such that $k^{\prime}(\lambda)>0$ on $\left(0, \lambda_{1}\right), k^{\prime}(\lambda)<0$ on $\left(\lambda_{1},+\infty\right)$ and $k^{\prime}\left(\lambda_{1}\right)=0$. Since $\lim _{\lambda \rightarrow 0^{+}} k(\lambda)=$ $\|\nabla u\|_{p}^{p}>0, \lim _{\lambda \rightarrow+\infty} k(\lambda)=-\infty$, there exists one $\lambda_{*}>0$ such that $k\left(\lambda_{*}\right)=0$. So the statements of $(2)$ and (3) can be shown easily. The last property is only a simply corollary of the fact $I(\lambda u)=\lambda j^{\prime}(\lambda)$. The proof of the lemma is complete.

Now, Lemma 2.2(4) shows that the Nehari manifold $\mathcal{N}$ is not empty. So the depth $d$ defined by 2.1) is meaningful. From 2.2 and the definition of $\mathcal{N}$ and $d$, it follows that $d \geq 0$. In the following lemma, we prove that $d$ is positive and is obtained by some $u \in \mathcal{N}$.

Lemma 2.3. $d$ is positive and there is a positive function $u \in \mathcal{N}$ such that $J(u)=d$.

Proof. Now, we only need to prove that there is a positive function $u \in \mathcal{N}$ such that $J(u)=d$. Let $\left\{u_{k}\right\}_{k=1}^{\infty} \subset \mathcal{N}$ be a minimizing sequence for $J$. That is,

$$
\lim _{k \rightarrow \infty} J\left(u_{k}\right)=d
$$

It is simple to show that $\left\{\left|u_{k}\right|\right\}_{k=1}^{\infty} \subset \mathcal{N}$ is also a minimizing sequence for $J$. Thus, without loss of generality, we assume that $u_{k}>0$ a.e. in $\Omega$ for all $k \in \mathcal{N}$. Since $\left\{J\left(u_{k}\right)\right\}_{k=1}^{\infty}$ is bounded and $I\left(u_{k}\right)=0$, by 2.2 , we infer that $\left\{u_{k}\right\}_{k=1}^{\infty}$ is bounded in $W_{0}^{1, p}(\Omega)$. Let $\mu>0$ be sufficiently small such that $q+\mu<p^{*}=n p /(n-p)$. Then the embedding 
$W_{0}^{1, p}(\Omega) \hookrightarrow L^{q+\mu}(\Omega)$ is compact, so there exist a function $u$ and a subsequence $\left\{u_{k_{i}}\right\}_{i=1}^{\infty}$ of $\left\{u_{k}\right\}_{k=1}^{\infty}$ such that

$$
\begin{array}{ll}
u_{k_{i}} \rightarrow u & \text { weakly in } W_{0}^{1, p}(\Omega), \\
u_{k_{i}} \rightarrow u & \text { strongly in } L^{q+\mu}(\Omega), \\
u_{k_{i}} \rightarrow u & \text { a.e. in } \Omega .
\end{array}
$$

Thus, $u \geq 0$ a.e. in $\Omega$. By the dominated convergence theorem, we have

$$
\begin{aligned}
\int_{\Omega}|u|^{q} \log |u| d x & =\lim _{i \rightarrow \infty} \int_{\Omega}\left|u_{k_{i}}\right|^{q} \log \left|u_{k_{i}}\right| d x, \\
\int_{\Omega}|u|^{q} d x & =\lim _{i \rightarrow \infty} \int_{\Omega}\left|u_{k_{i}}\right|^{q} d x .
\end{aligned}
$$

By the weak lower semicontinuity of $\|\cdot\|_{1, p}$, we have

$$
\|\nabla u\|_{p} \leq \liminf _{i \rightarrow \infty}\left\|\nabla u_{k_{i}}\right\|_{p}
$$

Combining (2.3)-(2.5) and the definition of $J(u)$, it follows that

$$
J(u) \leq \liminf _{i \rightarrow \infty} J\left(u_{k_{i}}\right)=d
$$

Combining (2.3), 2.5 and the definition of $I(u)$, we get

$$
I(u) \leq \liminf _{i \rightarrow \infty} I\left(u_{k_{i}}\right)=0
$$

Since $u_{k_{i}} \in \mathcal{N}$, we have $I\left(u_{k_{i}}\right)=0$. So, by using the fact $x^{-\mu} \log x \leq(e \mu)^{-1}$ for $x \geq 1$ and the Sobolev embedding inequality, we obtain

$$
\begin{aligned}
\left\|\nabla u_{k_{i}}\right\|_{p}^{p} & =\int_{\Omega}\left|u_{k_{i}}\right|^{q} \log \left|u_{k_{i}}\right| d x \leq \int_{\left\{x \in \Omega:\left|u_{k_{i}}\right| \geq 1\right\}}\left|u_{k_{i}}\right|^{q} \log \left|u_{k_{i}}\right| d x \\
& \leq(e \mu)^{-1} \int_{\left\{x \in \Omega:\left|u_{k_{i}}\right| \geq 1\right\}}\left|u_{k_{i}}\right|^{q+\mu} d x \leq(e \mu)^{-1}\left\|u_{k_{i}}\right\|_{q+\mu}^{q+\mu} \leq C(e \mu)^{-1}\left\|\nabla u_{k_{i}}\right\|_{p}^{q+\mu}
\end{aligned}
$$

where $C$ is the Sobolev constant satisfying $\left\|u_{k_{i}}\right\|_{q+\mu} \leq C\left\|\nabla u_{k_{i}}\right\|_{p}$. This implies that

$$
\int_{\Omega}\left|u_{k_{i}}\right|^{q} \log \left|u_{k_{i}}\right| d x=\left\|\nabla u_{k_{i}}\right\|_{p}^{p} \geq\left(\frac{e \mu}{C}\right)^{\frac{p}{q+\mu-p}} .
$$

Combining the above inequality with $(2.3)$, we get

$$
\int_{\Omega}|u|^{q} \log |u| d x \geq\left(\frac{e \mu}{C}\right)^{\frac{p}{q+\mu-p}} .
$$

Thus, we have $u \neq 0$. 
If $I(u)<0$, then by Lemma 2.2 , there exist a $\lambda_{*}$ such that $0<\lambda_{*}<1$ and $I\left(\lambda_{*} u\right)=0$. Therefore, we have

$$
\begin{aligned}
d \leq J\left(\lambda_{*} u\right) & =\left(\frac{1}{p}-\frac{1}{q}\right)\left\|\nabla\left(\lambda_{*} u\right)\right\|_{p}^{p}+\frac{1}{q^{2}}\left\|\lambda_{*} u\right\|_{q}^{q} \\
& =\lambda_{*}^{p}\left\{\left(\frac{1}{p}-\frac{1}{q}\right)\|\nabla u\|_{p}^{p}+\frac{1}{q^{2}}\|u\|_{q}^{q}\right\} \\
& \leq \lambda_{*}^{p} \liminf _{i \rightarrow \infty}\left\{\left(\frac{1}{p}-\frac{1}{q}\right)\left\|\nabla u_{k_{i}}\right\|_{p}^{p}+\frac{1}{q^{2}}\left\|u_{k_{i}}\right\|_{q}^{q}\right\} \\
& =\lambda_{*}^{p} \liminf _{i \rightarrow \infty} J\left(u_{k_{i}}\right) \\
& =\lambda_{*}^{p} d
\end{aligned}
$$

Since $\lambda_{*}^{p}<1$, the above formula contradicts the fact that $d>0$. Then, by (2.7), we have $I(u)=0$. So, $u \in \mathcal{N}$. From $(2.6)$ and the definition of $d$, we obtain $J(u)=d$.

Theorem 2.4. Let $2<p<q<p(1+2 / n)$, if $\left(u_{0}, u_{1}\right) \in W^{+}$, then the problem (1.1) has a global weak solution $u \in L^{\infty}\left(0, T ; W_{0}^{1, p}(\Omega)\right)$ with $u_{t} \in L^{2}\left(0, T ; H_{0}^{1}(\Omega)\right)$ and $\left(u, u_{t}\right) \in W^{+}$ for $0 \leq t<\infty$. Meanwhile, $u$ satisfies the energy inequality

$$
E(t)+\int_{0}^{t}\left\|\nabla u_{t}\right\|_{2}^{2} d \tau \leq E(0), \quad 0 \leq t<\infty .
$$

Furthermore, there exists a positive constant $\mathcal{K}_{0}$ such that the energy functional $E(t)$ satisfies the following polynomial decay estimate

$$
E(t) \leq \frac{\mathcal{K}_{0}}{1+t} \quad \text { for all } t \in[0,+\infty)
$$

In particular, if $E(0)<\min \left\{d, \frac{q-p}{p q}\left(\frac{e \mu}{C^{q+\mu}}\right)^{\frac{p}{q+\mu-p}}\right\}, I\left(u_{0}\right) \geq 0$ and $0<\mu<p^{*}-q$, then there exist positive constants $\mathcal{K}_{1}$ and $\mathcal{K}_{2}$, such that the $E(t)$ satisfies the exponential decay estimate as follows:

$$
E(t) \leq \mathcal{K}_{1} e^{-\mathcal{K}_{2} t} \quad \text { for all } t \in[0,+\infty)
$$

Theorem 2.5. Assume that $\left(u_{0}, u_{1}\right) \in W^{-}$and $2<q<p^{*}$, then the weak solution $u$ of the problem (1.1) blows up in finite time, that is, there exists a $T^{*}>0$ such that

$$
\lim _{t \rightarrow T^{*-}}\left(\|u\|_{2}^{2}+\int_{0}^{t}\|\nabla u\|_{2}^{2} d \tau\right)=+\infty .
$$

Moreover, the upper bound for blow up time $T^{*}$ can be estimated by

$$
T^{*} \leq \frac{2 b T_{0}^{2}+2\left\|u_{0}\right\|_{2}^{2}}{(q-2)\left(b T_{0}+\int_{\Omega} u_{0} u_{1} d x\right)-2\left\|\nabla u_{0}\right\|_{2}^{2}},
$$

where $b$ and $T_{0}$ are defined in 4.5 and 4.6. 
Theorem 2.6. Assume that all the conditions of Theorem 2.5 hold and $2<q<1+$ $n /(n-p)$, then the weak solution $u$ of the problem (1.1) become unbounded at finite time $t=T^{*}$ with

$$
\lim _{t \rightarrow T^{*-}}\left\|u_{t}\right\|_{2}^{2}+\|\nabla u\|_{p}^{p}=+\infty .
$$

Moreover, the lower bound for blow up time $T^{*}$ can be estimated by

$$
T^{*} \geq \int_{F(0)}^{\infty} \frac{d y}{y+\frac{1}{2}(e(q-1))^{-2}|\Omega|+\frac{1}{2}(e \mu)^{-2}|\Omega|+\frac{1}{2}(e \mu)^{-2} C^{p(q-1+\mu)} p^{q-1+\mu} y^{q-1+\mu}},
$$

where $0<\mu<(2 n-p) /(n-p)-q$ and $F(0)=\frac{1}{2}\left\|u_{1}\right\|_{2}^{2}+\frac{1}{p}\left\|\nabla u_{0}\right\|_{p}^{p}$.

\section{Global existence and energy decay estimate}

In this section, we focus on proving Theorem 2.4, which is divided into 5 steps.

Step 1: Global existence for the case of $\left(u_{0}, u_{1}\right) \in W_{1}^{+}$. By the definition of $E(t)$ and 2.2 , we know that $0 \leq J\left(u_{0}\right) \leq E(0)<d$, then we have respectively

(i) If $E(0)=0$ and $I\left(u_{0}\right) \geq 0$, then this implies that $\left(u_{0}, u_{1}\right)=(0,0)$, which is trivial case;

(ii) If $0<E(0)<d$ and $I\left(u_{0}\right)=0$, then it contradicts with the definition of potential depth $d$.

Therefore, we only need to consider the case of $0<E(0)<d$ and $I\left(u_{0}\right)>0$.

First, we select $\left\{w_{j}(x)\right\}$ as the orthogonal basis of $W_{0}^{1, p}(\Omega)$ and construct the approximate solution $u_{m}(x, t)$ of problem (1.1) as follows:

$$
u_{m}(x, t)=\sum_{j=1}^{m} g_{j}^{m}(t) w_{j}(x), \quad m=1,2, \ldots
$$

which satisfy

$$
\begin{gathered}
\left(u_{m t}, w_{j}\right)+\int_{0}^{t}\left(\left|\nabla u_{m}\right|^{p-2} \nabla u_{m}, \nabla w_{j}\right) d \tau+\left(\nabla u_{m}, \nabla w_{j}\right) \\
=\int_{0}^{t}\left(\left|u_{m}\right|^{q-2} u_{m} \log \left|u_{m}\right|, w_{j}\right) d \tau+\left(u_{1 m}, w_{j}\right)+\left(\nabla u_{0 m}, \nabla w_{j}\right) \quad \text { for } j=1,2, \ldots, m, \\
u_{0 m}=u_{m}(x, 0)=\sum_{j=1}^{m} g_{j}^{m}(0) w_{j}(x) \rightarrow u_{0} \quad \text { strongly in } W_{0}^{1, p}(\Omega), \\
u_{1 m}=u_{m t}(x, 0)=\sum_{j=1}^{m} g_{j t}^{m}(0) w_{j}(x) \rightarrow u_{1} \quad \text { strongly in } L^{2}(\Omega) .
\end{gathered}
$$


Now, differentiating of (3.1) with respect to $t$, multiplying the obtained equation by $g_{j t}^{m}(t)$, summing for $j$, and integrating over $[0, t]$, we can compute

$$
E\left(u_{m}, u_{m t}\right)+\int_{0}^{t}\left\|\nabla u_{m t}\right\|_{2}^{2} d \tau=E\left(u_{0 m}, u_{1 m}\right)<d, \quad 0 \leq t<+\infty
$$

for sufficiently large $m$.

Next, we will show that

$$
\left(u_{m}, u_{m t}\right) \in W_{1}^{+}, \quad 0 \leq t<+\infty
$$

for sufficiently large $m$. Arguing by contradiction, if it is false, then there exists $t_{*} \in$ $[0,+\infty)$ such that $\left(u_{m}\left(t_{*}\right), u_{m t}\left(t_{*}\right)\right) \in \partial W_{1}^{+}$, so

$$
E\left(u_{m}\left(t_{*}\right), u_{m t}\left(t_{*}\right)\right)=d,
$$

or

$$
I\left(u_{m}\left(t_{*}\right)\right)=0
$$

Nevertheless, it is clear that (3.6) could not occur by (3.4). If (3.7) holds, then by the definition of $d$, we have

$$
E\left(u_{m}\left(t_{*}\right)\right)>J\left(u_{m}\left(t_{*}\right)\right) \geq d,
$$

which is also contradictive with (3.4). Therefore, (3.5) is valid. Thus, $\left(u_{m}, u_{m t}\right) \in W_{1}^{+}$ such that

$$
d>E\left(u_{m}, u_{m t}\right)>J\left(u_{m}\right)=\frac{1}{q} I\left(u_{m}\right)+\left(\frac{1}{p}-\frac{1}{q}\right)\left\|\nabla u_{m}\right\|_{p}^{p}+\frac{1}{q^{2}}\left\|u_{m}\right\|_{q}^{q}
$$

for sufficiently large $m$ and $0 \leq t<+\infty$. So it follows that

$$
\begin{gathered}
\left\|\nabla u_{m}\right\|_{p}^{p}<\frac{p q d}{q-p}, \quad\left\|u_{m}\right\|_{q}^{q}<q^{2} d, \\
\int_{0}^{t}\left\|\nabla u_{m t}\right\|_{2}^{2} d \tau<d .
\end{gathered}
$$

By (3.8), (3.9), there exist functions $u, \chi$ and a subsequence of $\left\{u_{m}\right\}_{m=1}^{\infty}$ which we still denote it by $\left\{u_{m}\right\}_{m=1}^{\infty}$ such that

$$
\begin{aligned}
u_{m} \stackrel{*}{\rightarrow} u & \text { weakly star in } L^{\infty}\left(0, \infty ; W_{0}^{1, p}(\Omega)\right), \\
u_{m t} \rightarrow u_{t} & \text { weakly in } L^{2}\left(0, \infty ; H_{0}^{1}(\Omega)\right), \\
\left|\nabla u_{m}\right|^{p-2} \nabla u_{m} \stackrel{*}{\rightarrow} \chi & \text { weakly star in } L^{\infty}\left(0, \infty ; W_{0}^{-1, p^{\prime}}(\Omega)\right) .
\end{aligned}
$$

By Aubin-Lions-Simon Lemma (see [22, Corollary 4]), we get

$$
u_{m} \rightarrow u \quad \text { strongly in } C\left(0, \infty ; L^{2}(\Omega)\right) \text {, }
$$


so $u_{m} \rightarrow u$ a.e. $(x, t) \in \Omega \times[0, \infty), m \rightarrow+\infty$. This implies

$$
\left|u_{m}\right|^{q-2} u_{m} \log \left(\left|u_{m}\right|\right) \rightarrow|u|^{q-2} u \log (|u|) \quad \text { a.e. }(x, t) \in \Omega \times[0,+\infty) .
$$

Furthermore, since $2<q<p(1+2 / n)<p^{*}$, we can choose $\mu>0$ such that $(q-1+\mu) q^{\prime}<$ $p^{*}$, where $q^{\prime}=q /(q-1)$. Therefore, through direct calculation, we get

$$
\begin{aligned}
\int_{\Omega}\left|\phi_{m}\right|^{q^{\prime}} d x & =\int_{\left\{x \in \Omega:\left|u_{m}\right|<1\right\}}\left|\phi_{m}\right|^{q^{\prime}} d x+\int_{\left\{x \in \Omega:\left|u_{m}\right| \geq 1\right\}}\left|\phi_{m}\right|^{q^{\prime}} d x \\
& \leq(e(q-1))^{-q^{\prime}}|\Omega|+(e \mu)^{-q^{\prime}} \int_{\left\{x \in \Omega:\left|u_{m}\right| \geq 1\right\}}\left|u_{m}\right|^{(q-1+\mu) q^{\prime}} d x \\
& \leq(e(q-1))^{-q^{\prime}}|\Omega|+(e \mu)^{-q^{\prime}} C^{(q-1+\mu) q^{\prime}}\left\|\nabla u_{m}\right\|_{p}^{(q-1+\mu) q^{\prime}} \\
& \leq(e(q-1))^{-q^{\prime}}|\Omega|+(e \mu)^{-q^{\prime}} C^{(q-1+\mu) q^{\prime}}\left(\frac{p q d}{q-p}\right)^{\frac{(q-1+\mu) q^{\prime}}{p}}
\end{aligned}
$$

where $\phi_{m}=\left|u_{m}\right|^{q-1} \log \left|u_{m}\right|, C$ is the optimal Sobolev constant satisfying $\left\|u_{m}\right\|_{(q-1+\mu) q^{\prime}} \leq$ $C\left\|\nabla u_{m}\right\|_{p}$. Hence, from (3.13), (3.14) and Lions Lemma (see [13, Lemma 1.3, p. 12]), we have

$$
\left|u_{m}\right|^{q-2} u_{m} \log \left|u_{m}\right| \rightarrow|u|^{q-2} u \log |u| \quad \text { weakly star in } L^{\infty}\left(0, \infty ; L^{q^{\prime}}(\Omega)\right) .
$$

By $3.10-3.12$ and $(3.15)$, passing to the limit in 3.1$)-(3.3)$ as $m \rightarrow+\infty$, it follows that $u$ satisfies the initial condition $u(x, 0)=u_{0}$ in $W_{0}^{1, p}(\Omega), u_{t}(x, 0)=u_{1}$ in $L^{2}(\Omega)$, and

$$
\left(u_{t}, w\right)+\int_{0}^{t}(\chi, \nabla w) d \tau+(\nabla u, \nabla w)=\left(u_{1}, w\right)+\left(\nabla u_{0}, \nabla w\right)+\int_{0}^{t}\left(|u|^{q-2} u \log |u|, w\right) d \tau
$$

for all $w \in W_{0}^{1, p}(\Omega)$ and for a.e. $t \in[0,+\infty)$.

Finally, by the method of Browder and Minty in the theory of monotone operators, we obtain

$$
\chi=|\nabla u|^{p-2} \nabla u
$$

which implies

$$
\begin{aligned}
& \left(u_{t}, w\right)+\int_{0}^{t}\left(|\nabla u|^{p-2} \nabla u, \nabla w\right) d \tau+(\nabla u, \nabla w) \\
= & \left(u_{1}, w\right)+\left(\nabla u_{0}, \nabla w\right)+\int_{0}^{t}\left(|u|^{q-2} u \log |u|, w\right) d \tau
\end{aligned}
$$

for all $w \in W_{0}^{1, p}(\Omega)$ and for a.e. $t \in[0,+\infty)$.

Finally, since $\left(u_{0}, u_{1}\right) \in W_{1}^{+}$, by a standard contradiction argument it is easy to show that $\left(u, u_{t}\right) \in W_{1}^{+}$for $0 \leq t<+\infty$.

Step 2: Global existence for the case of $\left(u_{0}, u_{1}\right) \in W_{2}^{+}$. We will prove this step by considering two cases $E(0)=d, I\left(u_{0}\right)=0$ and $E(0)=d, I\left(u_{0}\right)>0$. 
(1) $E(0)=d, I\left(u_{0}\right)=0$ : From the definition of $d$, we have $J\left(u_{0}\right) \geq d$. However,

$$
\frac{1}{2}\left\|u_{1}\right\|_{2}^{2}+J\left(u_{0}\right)=E(0)=d,
$$

it follows that $J\left(u_{0}\right)<d$. So Case (1) is impossible.

(2) $E(0)=d, I\left(u_{0}\right)>0$ : In order to prove the global existence result of problem (1.1), we first choose a sequence $\left\{\gamma_{m}\right\}_{m=1}^{\infty} \subset(0,1)$ such that $\lim _{m \rightarrow \infty} \gamma_{m}=1$. Then we consider the following problem

$$
\begin{cases}u_{t t}-\Delta_{p} u-\Delta u_{t}=|u|^{q-2} u \log u, & (x, t) \in \Omega \times(0, T), \\ u(x, t)=0, & (x, t) \in \partial \Omega \times(0, T), \\ u(x, 0)=u_{0 m}, \quad u_{t}(x, 0)=u_{1 m}, & x \in \Omega,\end{cases}
$$

where $u_{0 m}=\gamma_{m} u_{0}, u_{1 m}=\gamma_{m} u_{1}$. Since $I\left(u_{0}\right)>0$, it follows from Lemma 2.2 that $\lambda_{*}>1$. Hence, we get

$$
I\left(u_{0 m}\right)=I\left(\gamma_{m} u_{0}\right)>0, \quad J\left(u_{0 m}\right)=J\left(\gamma_{m} u_{0}\right)<J\left(u_{0}\right),
$$

and

$$
0<E\left(u_{0 m}, u_{1 m}\right)=\frac{1}{2}\left\|u_{1 m}\right\|_{2}^{2}+J\left(u_{0 m}\right)<\frac{1}{2}\left\|u_{1}\right\|_{2}^{2}+J\left(u_{0}\right)=E(0)=d .
$$

Using the similar arguments as previous Step 1, we find that problem (3.16) admits a global weak solution $u_{m}$ which satisfies

$$
u_{m} \in L^{\infty}\left(0, \infty ; W_{0}^{1, p}(\Omega)\right), \quad u_{m t} \in L^{2}\left(0, \infty ; H_{0}^{1}(\Omega)\right)
$$

with initial data

$$
\begin{aligned}
u_{m}(x, 0)=u_{0 m} \rightarrow u_{0} & \text { strongly in } W_{0}^{1, p}(\Omega), \\
u_{m t}(x, 0)=u_{1 m} \rightarrow u_{1} & \text { strongly in } L^{2}(\Omega),
\end{aligned}
$$

and

$$
\begin{aligned}
& \left(u_{m t}, v\right)+\int_{0}^{t}\left(\left|\nabla u_{m}\right|^{p-2} \nabla u_{m}, \nabla v\right) d \tau+\left(\nabla u_{m}, \nabla v\right) \\
= & \left(u_{1 m}, v\right)+\left(\nabla u_{0 m}, \nabla v\right)+\int_{0}^{t}\left(\left|u_{m}\right|^{q-2} u_{m} \log \left|u_{m}\right|, v\right) d \tau
\end{aligned}
$$

for any $v \in W_{0}^{1, p}(\Omega)$, and for a.e. $0 \leq t<\infty$.

The remainder of the proof can be processed similarly as previous Step 1. We derive

$$
\begin{aligned}
& \left(u_{t}, v\right)+\int_{0}^{t}\left(|\nabla u|^{p-2} \nabla u, \nabla v\right) d \tau+(\nabla u, \nabla v) \\
= & \left(u_{1}, v\right)+\left(\nabla u_{0}, \nabla v\right)+\int_{0}^{t}\left(|u|^{q-2} u \log |u|, v\right) d \tau
\end{aligned}
$$


for fixed $v \in W_{0}^{1, p}(\Omega)$, for a.e. $0 \leq t<\infty$. Hence, $u$ is a global weak solution for problem (1.1). From $\left(u_{0}, u_{1}\right) \in W_{2}^{+}$, by a standard contradiction argument it is easy to show that $\left(u, u_{t}\right) \in W_{2}^{+}$for $0 \leq t<+\infty$.

Step 3: Energy inequality. Now, we show that the weak solution satisfies the energy inequality 2.8). First, we prove

$$
\lim _{m \rightarrow \infty} \int_{\Omega}\left|u_{m}\right|^{q} \log \left|u_{m}\right| d x=\int_{\Omega}|u|^{q} \log |u| d x,
$$

and

$$
\lim _{m \rightarrow \infty} \int_{\Omega}\left|u_{m}\right|^{q} d x=\int_{\Omega}|u|^{q} d x .
$$

In fact, by Hölder inequality, for each fixed $t>0$, we have

$$
\begin{aligned}
& \left.\left|\int_{\Omega}\right| u_{m}\right|^{q} \log \left|u_{m}\right| d x-\int_{\Omega}|u|^{q} \log |u| d x \mid \\
\leq & \left.\int_{\Omega}|| u_{m}\right|^{q} \log \left|u_{m}\right|-|u|^{q} \log |u| \mid d x \\
\leq & \left.\int_{\Omega}|q| \varphi_{1}\right|^{q-1} \log \left|\varphi_{1}\right|+\left|\varphi_{1}\right|^{q-1}|| u-u_{m} \mid d x \\
\leq & q\left(\left.\int_{\Omega}|| \varphi_{1}\right|^{q-1} \log \left|\varphi_{1}\right|^{q^{\prime}} d x\right)^{1 / q^{\prime}}\left\|u-u_{m}\right\|_{q}+\left\|\varphi_{1}\right\|_{q}^{q-1}\left\|u-u_{m}\right\|_{q} \\
\leq & C\left\|u-u_{m}\right\|_{q} \rightarrow 0,
\end{aligned}
$$

and

$$
\begin{aligned}
\left.\left|\int_{\Omega}\right| u_{m}\right|^{q} d x-\int_{\Omega}|u|^{q} d x \mid & \leq\left.\int_{\Omega}|| u_{m}\right|^{q}-\left.|u|^{q}\left|d x \leq q \int_{\Omega}\right| \varphi_{2}\right|^{q-1}\left|u-u_{m}\right| d x \\
& \leq q\left\|\varphi_{2}\right\|_{q}^{q-1}\left\|u-u_{m}\right\|_{q} \leq C\left\|u-u_{m}\right\|_{q} \rightarrow 0,
\end{aligned}
$$

where $\varphi_{i}=u+\theta_{i} u_{m}, 0<\theta_{i}<1(i=1,2)$.

On the other hand, making use of Fatou Lemma and (3.4), we deduce

$$
\begin{aligned}
& \frac{1}{2}\left\|u_{t}\right\|_{2}^{2}+\frac{1}{p}\|\nabla u\|_{p}^{p}+\int_{0}^{t}\left\|\nabla u_{t}\right\|_{2}^{2} d \tau \\
\leq & \frac{1}{2} \liminf _{m \rightarrow \infty}\left\|u_{m t}\right\|_{2}^{2}+\frac{1}{p} \liminf _{m \rightarrow \infty}\left\|\nabla u_{m}\right\|_{p}^{p}+\liminf _{m \rightarrow \infty} \int_{0}^{t}\left\|\nabla u_{m t}\right\|_{2}^{2} d \tau \\
= & \liminf _{m \rightarrow \infty}\left[E\left(u_{0 m}, u_{1 m}\right)+\frac{1}{q} \int_{\Omega}\left|u_{m}\right|^{q} \log \left|u_{m}\right| d x-\frac{1}{q^{2}} \int_{\Omega}\left|u_{m}\right|^{q} d x\right] \\
= & E(0)+\frac{1}{q} \int_{\Omega}|u|^{q} \log |u| d x-\frac{1}{q^{2}} \int_{\Omega}|u|^{q} d x .
\end{aligned}
$$

Hence, (2.8) hold. 
Step 4: Polynomial decay estimate of energy for the case of $\left(u_{0}, u_{1}\right) \in W^{+}$. Since $\left(u_{0}, u_{1}\right) \in W^{+}$, a standard contradiction argument shows $\left(u, u_{t}\right) \in W^{+}$for each $t$. First, from the definition of energy functional $E(t),(2.2)$ and $I(u) \geq 0$, we derive

$$
\begin{aligned}
E(0) & \geq E(t)+\int_{0}^{t}\left\|\nabla u_{t}\right\|_{2}^{2} d \tau \\
& =\frac{1}{2}\left\|u_{t}\right\|_{2}^{2}+\frac{1}{q} I(u)+\frac{q-p}{p q}\|\nabla u\|_{p}^{p}+\frac{1}{q^{2}}\|u\|_{q}^{q}+\int_{0}^{t}\left\|\nabla u_{t}\right\|_{2}^{2} d \tau \\
& \geq \frac{1}{2}\left\|u_{t}\right\|_{2}^{2}+\frac{q-p}{p q}\|\nabla u\|_{p}^{p}+\frac{1}{q^{2}}\|u\|_{q}^{q}+\int_{0}^{t}\left\|\nabla u_{t}\right\|_{2}^{2} d \tau .
\end{aligned}
$$

A combination of $(3.17)$ and $E(0) \leq d$, we get

$$
\int_{0}^{t}\left\|u_{t}\right\|_{2}^{2} d \tau \leq \frac{1}{\lambda_{1}} \int_{0}^{t}\left\|\nabla u_{t}\right\|_{2}^{2} d \tau \leq \frac{d}{\lambda_{1}},
$$

where $\lambda_{1}$ is the first eigenvalue of the following problem

$$
\begin{cases}-\Delta \phi(x)=\lambda \phi(x), & x \in \Omega \\ \phi(x)=0, & x \in \partial \Omega\end{cases}
$$

for $\phi(x) \in H_{0}^{1}(\Omega)$.

Next, multiplying the first equation of problem (1.1) by $u$ and integrating over $\Omega \times(0, t)$. Using Young inequality, we have

$$
\begin{aligned}
\int_{0}^{t} I(u) d \tau & =-\int_{0}^{t}\left(u_{t t}, u\right) d \tau-\int_{0}^{t}\left(\nabla u_{t}, \nabla u\right) d \tau \\
& =-\int_{\Omega} u_{t} u d x+\int_{\Omega} u_{1} u_{0} d x+\int_{0}^{t}\left\|u_{t}\right\|_{2}^{2} d \tau+\frac{1}{2}\left\|\nabla u_{0}\right\|_{2}^{2}-\frac{1}{2}\|\nabla u\|_{2}^{2} \\
& \leq \frac{1}{2}\|u\|_{H_{0}^{1}}^{2}+\frac{1}{2}\left\|u_{t}\right\|_{2}^{2}+\int_{0}^{t}\left\|u_{t}\right\|_{2}^{2} d \tau+\frac{1}{2}\left\|u_{1}\right\|_{2}^{2}+\frac{1}{2}\left\|u_{0}\right\|_{H_{0}^{1}}^{2} \\
& \leq \frac{C^{2}}{2}\|u\|_{W^{1, p}}^{2}+\frac{1}{2}\left\|u_{t}\right\|_{2}^{2}+\int_{0}^{t}\left\|u_{t}\right\|_{2}^{2} d \tau+\frac{1}{2}\left\|u_{1}\right\|_{2}^{2}+\frac{1}{2}\left\|u_{0}\right\|_{H_{0}^{1}}^{2}
\end{aligned}
$$

where $C$ stands for the best constant in the embedding $W^{1, p}(\Omega) \hookrightarrow H_{0}^{1}(\Omega)$. Using (3.17), (3.18) and $u \in L^{\infty}\left(0, T ; W_{0}^{1, p}(\Omega)\right)$, then 3.19 implies that

$$
\int_{0}^{t} I(u) d \tau \leq C \quad \text { for } 0<t<+\infty .
$$

From $I(u) \geq 0$, we know that there exists a $\lambda_{*}>1$ such that $I\left(\lambda_{*} u\right)=0$. This implies

$$
\lambda_{*}^{q}\left\{\left(\frac{1}{p}-\frac{1}{q}\right)\|\nabla u\|_{p}^{p}+\frac{1}{q^{2}}\|u\|_{q}^{q}\right\} \geq J\left(\lambda_{*} u\right) \geq d .
$$


Combining (2.2) and 2.8, we have

$$
\left(\frac{1}{p}-\frac{1}{q}\right)\|\nabla u\|_{p}^{p}+\frac{1}{q^{2}}\|u\|_{q}^{q} \leq J(u)<E(t) \leq E(0) \leq d .
$$

It follows from 3.21 and 3.22 that

$$
\lambda_{*}>\left(\frac{d}{E(0)}\right)^{1 / q}>1 .
$$

On the other hand, we have

$$
\begin{aligned}
0=I\left(\lambda_{*} u\right) & =\lambda_{*}^{p}\|\nabla u\|_{p}^{p}-\lambda_{*}^{q} \int_{\Omega}|u|^{q} \log |u| d x-\lambda_{*}^{q} \log \lambda_{*}\|u\|_{q}^{q} \\
& =\lambda_{*}^{q} I(u)-\left(\lambda_{*}^{q}-\lambda_{*}^{p}\right)\|\nabla u\|_{p}^{p}-\lambda_{*}^{q} \log \lambda_{*}\|u\|_{q}^{q}
\end{aligned}
$$

Hence, we obtain

$$
I(u)=\left(1-\frac{1}{\lambda_{*}^{q-p}}\right)\|\nabla u\|_{p}^{p}+\log \lambda_{*}\|u\|_{q}^{q} .
$$

Combining the above equation with 3.20 , we obtain

$$
\int_{0}^{t}\|\nabla u\|_{p}^{p} d \tau \leq C
$$

and

$$
\int_{0}^{t}\|u\|_{q}^{q} d \tau \leq C
$$

Differentiating $E(t)$ and using (1.1), we can compute

$$
E^{\prime}(t)=-\int_{\Omega}\left|\nabla u_{t}\right|^{2} d x \leq 0 .
$$

Since

$$
\frac{d}{d t}[(1+t) E(t)]=(1+t) E^{\prime}(t)+E(t) \leq E(t),
$$

then integrating 3.25 over $(0, t)$, we can have

$$
\begin{aligned}
(1+t) E(t) \leq & E(0)+\int_{0}^{t} E(t) d \tau \\
= & E(0)+\frac{1}{2} \int_{0}^{t}\left\|u_{t}\right\|_{2}^{2} d \tau+\frac{1}{q} \int_{0}^{t} I(u) d \tau+\left(\frac{1}{p}-\frac{1}{q}\right) \int_{0}^{t}\|\nabla u\|_{p}^{p} d \tau \\
& +\frac{1}{q^{2}} \int_{0}^{t}\|u\|_{q}^{q} d \tau .
\end{aligned}
$$


Thus, applying $E(0) \leq d, 3.18$, 3.20, 3.23) and (3.24) to $(3.26$, we can derive that there exists a positive constants $\mathcal{K}_{0}$ such that the energy functional $E(t)$ satisfies the following polynomial decay estimation

$$
E(t) \leq \frac{\mathcal{K}_{0}}{1+t} \quad \text { for all } t \in[0,+\infty)
$$

Step 5: Exponential decay estimate of energy for the case of $E(0)<\min \left\{d, \frac{q-p}{p q}\right.$ $\left.\times\left(\frac{e \mu}{C^{q+\mu}}\right)^{\frac{p}{q+\mu-p}}\right\}$ and $I\left(u_{0}\right) \geq 0$. We define

$$
L(t)=E(t)+\varepsilon \int_{\Omega} u u_{t} d x+\frac{\varepsilon}{2} \int_{\Omega}|\nabla u|^{2} d x
$$

where $\varepsilon$ is a positive constant to be specified later.

By the Young inequality, we can easily know that there exist two positive constant $\alpha_{1}$ and $\alpha_{2}$ such that

$$
\alpha_{1} E(t) \leq L(t) \leq \alpha_{2} E(t) \text { for all } t \in[0,+\infty)
$$

That is to say, $L(t)$ and $E(t)$ are equivalent.

Now, differentiating $L(t)$ and using (1.1), we have

$$
\begin{aligned}
L^{\prime}(t)= & E^{\prime}(t)+\varepsilon \int_{\Omega}\left|u_{t}\right|^{2} d x+\varepsilon \int_{\Omega} u u_{t t} d x+\varepsilon \int_{\Omega} \nabla u \nabla u_{t} d x \\
= & -\int_{\Omega}\left|\nabla u_{t}\right|^{2} d x+\varepsilon \int_{\Omega}\left|u_{t}\right|^{2} d x+\varepsilon \int_{\Omega} u\left[\Delta_{p} u+|u|^{q-2} u \log |u|\right] d x \\
= & -\int_{\Omega}\left|\nabla u_{t}\right|^{2} d x+\varepsilon \int_{\Omega}\left|u_{t}\right|^{2} d x-\varepsilon \int_{\Omega}|\nabla u|^{p} d x+\varepsilon \int_{\Omega}|u|^{q} \log |u| d x \\
= & -\beta \varepsilon E(t)+\frac{\beta \varepsilon}{2}\left\|u_{t}\right\|_{2}^{2}+\frac{\beta \varepsilon}{p}\|\nabla u\|_{p}^{p}-\frac{\beta \varepsilon}{q} \int_{\Omega}|u|^{q} \log |u| d x+\frac{\beta \varepsilon}{q^{2}}\|u\|_{q}^{q} \\
& -\int_{\Omega}\left|\nabla u_{t}\right|^{2} d x+\varepsilon \int_{\Omega}\left|u_{t}\right|^{2} d x-\varepsilon \int_{\Omega}|\nabla u|^{p} d x+\varepsilon \int_{\Omega}|u|^{q} \log |u| d x \\
\leq & -\beta \varepsilon E(t)+\left(\frac{\beta \varepsilon}{2}+\varepsilon-\lambda_{1}\right)\left\|u_{t}\right\|_{2}^{2}+\left(\frac{\beta \varepsilon}{p}-\varepsilon\right)\|\nabla u\|_{p}^{p} \\
& +\left(\varepsilon-\frac{\beta \varepsilon}{q}\right) \int_{\Omega}|u|^{q} \log |u| d x+\frac{\beta \varepsilon}{q^{2}}\|u\|_{q}^{q} .
\end{aligned}
$$

By virtue of the Sobolev embedding inequality and (3.17), we obtain

$$
\begin{aligned}
\int_{\Omega}|u|^{q} \log |u| d x & \leq \int_{\{x \in \Omega:|u| \geq 1\}}|u|^{q} \log |u| d x \leq(e \mu)^{-1} \int_{\{x \in \Omega:|u| \geq 1\}}|u|^{q+\mu} d x \\
& \leq(e \mu)^{-1}\|u\|_{q+\mu}^{q+\mu} \leq(e \mu)^{-1} C^{q+\mu}\|\nabla u\|_{p}^{q+\mu} \\
& \leq(e \mu)^{-1} C^{q+\mu}\left(\frac{p q E(0)}{q-p}\right)^{\frac{q+\mu-p}{p}}\|\nabla u\|_{p}^{p},
\end{aligned}
$$


and

$$
\|u\|_{q}^{q} \leq C^{q}\|\nabla u\|_{p}^{q} \leq C^{q}\left(\frac{p q E(0)}{q-p}\right)^{\frac{q-p}{p}}\|\nabla u\|_{p}^{p},
$$

where $C$ is the Sobolev constant satisfying $\|u\|_{q+\mu} \leq C\|\nabla u\|_{p},\|u\|_{q} \leq C\|\nabla u\|_{p}$. Inserting (3.29) and (3.30) into 3.28 , it follows that

$$
\begin{aligned}
L^{\prime}(t) \leq & -\beta \varepsilon E(t)+\left(\frac{\beta \varepsilon}{2}+\varepsilon-\lambda_{1}\right)\left\|u_{t}\right\|_{2}^{2} \\
& +\varepsilon\left\{\frac{\beta}{p}+\frac{C^{q+\mu}}{e \mu}\left(\frac{p q E(0)}{q-p}\right)^{\frac{q+\mu-p}{p}}-1-\frac{\beta}{q} \frac{C^{q+\mu}}{e \mu}\left(\frac{p q E(0)}{q-p}\right)^{\frac{q+\mu-p}{p}}\right. \\
& \left.+\frac{\beta}{q^{2}} C^{q}\left(\frac{p q E(0)}{q-p}\right)^{\frac{q-p}{p}}\right\}\|\nabla u\|_{p}^{p} .
\end{aligned}
$$

Since $E(0)<\frac{q-p}{p q}\left(\frac{e \mu}{C^{q+\mu}}\right)^{\frac{p}{q+\mu-p}}$, we get

$$
\frac{C^{q+\mu}}{e \mu}\left(\frac{p q E(0)}{q-p}\right)^{\frac{q+\mu-p}{p}}-1<0 .
$$

By choosing $\beta>0$ small enough such that

$$
\frac{\beta}{p}+\frac{C^{q+\mu}}{e \mu}\left(\frac{p q E(0)}{q-p}\right)^{\frac{q+\mu-p}{p}}+\frac{\beta}{q^{2}} C^{q}\left(\frac{p q E(0)}{q-p}\right)^{\frac{q-p}{p}}-1<0 .
$$

Then, choosing $\varepsilon$ small enough such that

$$
\frac{\beta \varepsilon}{2}+\varepsilon-\lambda_{1}<0
$$

Hence, a combination of (3.27) and (3.31) yields

$$
L^{\prime}(t) \leq-\beta \varepsilon E(t) \leq \frac{-\beta \varepsilon}{\alpha_{2}} L(t)
$$

Finally, integrating 3.32 over $(0, t)$, we can deduce that there exist $\mathcal{K}_{1}=L(0) / \alpha_{1}$ and $\mathcal{K}_{2}=\beta \varepsilon / \alpha_{2}$ such that

$$
E(t) \leq \mathcal{K}_{1} e^{-\mathcal{K}_{2} t} \quad \text { for all } t \in[0,+\infty)
$$

This completes the proof of Theorem 2.4.

4. Blow-up in the finite time

In this section, we mainly prove Theorems 2.5 and 2.6 . 
First, $\left(u_{0}, u_{1}\right) \in W^{-}$, by Faedo-Galerkin methods (see [4, 12, 13]), it is not difficult to obtain a local solution $u$ of problem (1.1) satisfying the energy inequality

$$
E(t)+\int_{0}^{t}\left\|\nabla u_{t}\right\|_{2}^{2} d \tau \leq E(0) \leq d, \quad 0 \leq t \leq T^{*},
$$

where $T^{*}$ is the maximal existence time of the solution $u$.

Next, we introduce the following lemmas which plays an important role in proving our main results.

Lemma 4.1. Assume that $\left(u_{0}, u_{1}\right) \in W^{-}$, then we have $\left(u, u_{t}\right) \in W^{-}$for any $t>0$.

Proof. To prove $\left(u, u_{t}\right) \in W^{-}$for any $t>0$. We suppose on the contrary that $\left(u, u_{t}\right) \notin W^{-}$ on $t=t_{0}$, then there exists a sequence $\left\{t_{n}\right\}, t_{n} \rightarrow t_{0}^{-}$, such that $I\left(u\left(t_{n}\right)\right)<0, E\left(u\left(t_{n}\right)\right) \leq d$. By the weak lower semicontinuity of $I\left(u\left(t_{n}\right)\right)$ and $E\left(u\left(t_{n}\right)\right)$ in $W_{0}^{1, p}(\Omega)$, we have

$$
I\left(u\left(t_{0}\right)\right) \leq \liminf _{n \rightarrow \infty} I\left(u\left(t_{n}\right)\right) \leq 0, \quad E\left(t_{0}\right) \leq \liminf _{n \rightarrow \infty} E\left(u\left(t_{n}\right)\right) \leq d
$$

By the assumption of $\left(u, u_{t}\right) \notin W^{-}$on $t=t_{0}$, then $I\left(u\left(t_{0}\right)\right) \geq 0$ or $E\left(u\left(t_{0}\right)\right)>d$.

Case 1. $I\left(u\left(t_{0}\right)\right)>0$ is impossible, because of the weak lower semicontinuity of $I\left(u\left(t_{n}\right)\right)$. If $I\left(u\left(t_{0}\right)\right)=0$, by the definition of $d, E(t)$ and $J(u)$, we can obtain

$$
d=\inf _{u \in N} J(u) \leq J\left(u\left(t_{0}\right)\right)<E\left(u\left(t_{0}\right)\right) \leq d .
$$

Obviously, the above formula is contradictory.

Case 2. $E\left(u\left(t_{0}\right)\right)>d$ is obviously impossible. This contradicts the weak lower semicontinuity of $E\left(u\left(t_{n}\right)\right)$ in $W_{0}^{1, p}(\Omega)$.

Thus, $\left(u, u_{t}\right) \in W^{-}$for any $t>0$.

Lemma 4.2. [8] Let $M(t)$ be a positive $C^{2}$ function, which satisfies, for $t>0$, inequality

$$
M(t) M^{\prime \prime}(t)-(1+\alpha)\left[M^{\prime}(t)\right]^{2} \geq 0
$$

for some $\alpha>0$. If $M(0)>0$ and $M^{\prime}(0)>0$, then there exists a time $T^{*} \leq M(0) /\left(\alpha M^{\prime}(0)\right)$ such that $\lim _{t \rightarrow T^{*-}} M(t)=\infty$.

Proof of Theorem 2.5. We define

$$
M(t)=\|u\|_{2}^{2}+\int_{0}^{t}\|\nabla u\|_{2}^{2} d \tau+(T-t)\left\|\nabla u_{0}\right\|_{2}^{2}+b\left(t+T_{0}\right)^{2},
$$

where $b$ and $T_{0}$ are positive constants, which will be described later. 
By calculating the first-order differential and second-order differential of $M(t)$, we obtain that

$$
\begin{aligned}
M^{\prime}(t) & =2 \int_{\Omega} u u_{t} d x+2 \int_{0}^{t} \int_{\Omega} \nabla u \nabla u_{t} d x d \tau+2 b\left(t+T_{0}\right), \\
M^{\prime \prime}(t) & =2 \int_{\Omega}\left|u_{t}\right|^{2} d x+2 \int_{\Omega} u_{t t} u d x-2 \int_{\Omega} u \Delta u_{t} d x+2 b \\
& =2 \int_{\Omega}\left|u_{t}\right|^{2} d x+2 \int_{\Omega} u\left[\Delta_{p} u+|u|^{q-2} u \log |u|\right] d x+2 b \\
& =2 \int_{\Omega}\left|u_{t}\right|^{2} d x-2 \int_{\Omega}|\nabla u|^{p} d x+2 \int_{\Omega}|u|^{q} \log |u| d x+2 b \\
& =2 \int_{\Omega}\left|u_{t}\right|^{2} d x-2 I(u)+2 b .
\end{aligned}
$$

Combining 4.2 with 4.3 , we get

$$
\begin{aligned}
& M(t) M^{\prime \prime}(t)-\frac{q+2}{4}\left[M^{\prime}(t)\right]^{2} \\
= & 2 M(t)\left(\int_{\Omega}\left|u_{t}\right|^{2} d x-\int_{\Omega}|\nabla u|^{p} d x+\int_{\Omega}|u|^{q} \log |u| d x+b\right) \\
& -(q+2)\left(\int_{\Omega} u u_{t} d x+\int_{0}^{t} \int_{\Omega} \nabla u \nabla u_{t} d x d \tau+b\left(t+T_{0}\right)\right)^{2} \\
= & 2 M(t)\left(\int_{\Omega}\left|u_{t}\right|^{2} d x-\int_{\Omega}|\nabla u|^{p} d x+\int_{\Omega}|u|^{q} \log |u| d x+b\right) \\
& +(q+2)\left[G(t)-\left(M(t)-(T-t)\left\|\nabla u_{0}\right\|_{2}^{2}\right)\left(\left\|u_{t}\right\|_{2}^{2}+\int_{0}^{t}\left\|\nabla u_{t}\right\|_{2}^{2} d \tau+b\right)\right],
\end{aligned}
$$

where

$$
\begin{aligned}
G(t)= & \left(\|u\|_{2}^{2}+\int_{0}^{t}\|\nabla u\|_{2}^{2} d \tau+b\left(t+T_{0}\right)^{2}\right)\left(\left\|u_{t}\right\|_{2}^{2}+\int_{0}^{t}\left\|\nabla u_{t}\right\|_{2}^{2} d \tau+b\right) \\
& -\left(\int_{\Omega} u u_{t} d x+\int_{0}^{t} \int_{\Omega} \nabla u \nabla u_{t} d x d \tau+b\left(t+T_{0}\right)\right)^{2} .
\end{aligned}
$$

Using Hölder inequality and Young's inequality, it is easy to testify that $G(t) \geq 0$. Therefore, by (4.4) and $G(t) \geq 0$, we can get the following inequality

$$
M(t) M^{\prime \prime}(t)-\frac{q+2}{4}\left[M^{\prime}(t)\right]^{2} \geq M(t) \xi(t),
$$

where

$$
\xi(t)=-q\left\|u_{t}\right\|_{2}^{2}-2\|\nabla u\|_{p}^{p}+2 \int_{\Omega}|u|^{q} \log |u| d x-(q+2) \int_{0}^{t}\left\|\nabla u_{t}\right\|_{2}^{2} d \tau-q b .
$$

By the definition of $E(t)$ and (4.1), we have

$$
\begin{aligned}
\xi(t) & =-2 q E(t)+\left(\frac{2 q}{p}-2\right)\|\nabla u\|_{p}^{p}+\frac{2}{q}\|u\|_{q}^{q}-(q+2) \int_{0}^{t}\left\|\nabla u_{t}\right\|_{2}^{2} d \tau-q b \\
& \geq-2 q d+\left(\frac{2 q}{p}-2\right)\|\nabla u\|_{p}^{p}+\frac{2}{q}\|u\|_{q}^{q}+(q-2) \int_{0}^{t}\left\|\nabla u_{t}\right\|_{2}^{2} d \tau-q b .
\end{aligned}
$$


Since $\left(u_{0}, u_{1}\right) \in W^{-}$and Lemma 4.1, we have $\left(u, u_{t}\right) \in W^{-}$for any $t>0$, i.e., $I(u)<0$. Using Lemma 2.2, then there exists a $\lambda_{*} \in(0,1)$ such that $I\left(\lambda_{*} u\right)=0$. Hence, by the definition of $d$ and $(2.2)$, the following formula holds

$$
\left(\frac{1}{p}-\frac{1}{q}\right)\|\nabla u\|_{p}^{p}+\frac{1}{q^{2}}\|u\|_{q}^{q} \geq\left(\frac{1}{p}-\frac{1}{q}\right) \lambda_{*}^{p}\|\nabla u\|_{p}^{p}+\frac{1}{q^{2}} \lambda_{*}^{q}\|u\|_{q}^{q}=J\left(\lambda_{*} u\right) \geq d,
$$

taking $b$ small enough, which satisfies

$$
0<b \leq \frac{-2 q d+\left(\frac{2 q}{p}-2\right)\|\nabla u\|_{p}^{p}+\frac{2}{q}\|u\|_{q}^{q}+(q-2) \int_{0}^{t}\left\|\nabla u_{t}\right\|_{2}^{2} d \tau}{q},
$$

thus, we can see that $\xi(t) \geq 0$.

Through the above discussion, we get that

$$
M(t) M^{\prime \prime}(t)-\frac{q+2}{4}\left[M^{\prime}(t)\right]^{2} \geq 0 .
$$

By the definition of $M(t), M(0)=\left\|u_{0}\right\|_{2}^{2}+T\left\|\nabla u_{0}\right\|_{2}^{2}+b T_{0}^{2}>0$, we choose $T_{0}$ large enough, which satisfies

$$
T_{0}>\frac{(q-2)\left(\left\|u_{0}\right\|_{2}^{2}+\left\|u_{1}\right\|_{2}^{2}\right)+4\left\|\nabla u_{0}\right\|_{2}^{2}}{2(q-2) b},
$$

thus, $M^{\prime}(0)=2 b T_{0}+2 \int_{\Omega} u_{0} u_{1} d x \geq 2 b T_{0}-\left\|u_{0}\right\|_{2}^{2}-\left\|u_{1}\right\|_{2}^{2}>0$.

According to Lemma $4.2, M(t) \rightarrow \infty\left(t \rightarrow T^{*}\right), T^{*}$ satisfying

$$
T^{*} \leq \frac{4 M(0)}{(q-2) M^{\prime}(0)}=\frac{2 b T_{0}^{2}+2\left\|u_{0}\right\|_{2}^{2}+2 T\left\|\nabla u_{0}\right\|_{2}^{2}}{(q-2)\left(b T_{0}+\int_{\Omega} u_{0} u_{1} d x\right)}
$$

that is,

$$
T^{*} \leq \frac{4 M(0)}{(q-2) M^{\prime}(0)}=\frac{2 b T_{0}^{2}+2\left\|u_{0}\right\|_{2}^{2}}{(q-2)\left(b T_{0}+\int_{\Omega} u_{0} u_{1} d x\right)-2\left\|\nabla u_{0}\right\|_{2}^{2}},
$$

$\lim _{t \rightarrow T^{*-}} M(t)=\infty$. This completes the proof of Theorem 2.5.

Proof of Theorem 2.6. We define the function

$$
F(t)=\frac{1}{2}\left\|u_{t}\right\|_{2}^{2}+\frac{1}{p}\|\nabla u\|_{p}^{p},
$$

by calculating the first-order differential of $F(t)$, we get

$$
\begin{aligned}
F^{\prime}(t) & =\int_{\Omega} u_{t} u_{t t} d x+\int_{\Omega}|\nabla u|^{p-2} \nabla u \nabla u_{t} d x \\
& =\int_{\Omega} u_{t}\left(\Delta u_{t}+|u|^{q-2} u \log |u|\right) d x \\
& =-\left\|\nabla u_{t}\right\|_{2}^{2}+\int_{\Omega} u_{t}|u|^{q-2} u \log |u| d x
\end{aligned}
$$


Due to $2<q<1+n /(n-p)$, we choose $\mu>0$ small enough such that $q-1+\mu<$ $n /(n-p)$. By the Young inequality and Sobolev inequality, we have

$$
\begin{aligned}
& \int_{\Omega} u_{t}|u|^{q-2} u \log |u| d x \\
\leq & \frac{1}{2} \int_{\Omega}\left|u_{t}\right|^{2} d x+\left.\frac{1}{2} \int_{\Omega}|| u\right|^{q-2} u \log |u|^{2} d x \\
= & \frac{1}{2}\left\|u_{t}\right\|_{2}^{2}+\left.\frac{1}{2} \int_{\{x \in \Omega:|u|<1\}}|| u\right|^{q-2} u \log |u|^{2} d x+\left.\frac{1}{2} \int_{\{x \in \Omega:|u| \geq 1\}}|| u\right|^{q-2} u \log |u|^{2} d x \\
\leq & \frac{1}{2}\left\|u_{t}\right\|_{2}^{2}+\frac{1}{2}(e(q-1))^{-2}|\Omega|+\frac{1}{2}(e \mu)^{-2} \int_{\{x \in \Omega:|u| \geq 1\}}|u|^{2(q-1+\mu)} d x \\
\leq & \frac{1}{2}\left\|u_{t}\right\|_{2}^{2}+\frac{1}{2}(e(q-1))^{-2}|\Omega|+\frac{1}{2}(e \mu)^{-2}|\Omega|+\frac{1}{2}(e \mu)^{-2} \int_{\Omega}|u|^{p(q-1+\mu)} d x \\
\leq & \frac{1}{2}\left\|u_{t}\right\|_{2}^{2}+\frac{1}{2}(e(q-1))^{-2}|\Omega|+\frac{1}{2}(e \mu)^{-2}|\Omega|+\frac{1}{2}(e \mu)^{-2} C^{p(q-1+\mu)}\|\nabla u\|_{p}^{p(q-1+\mu)} \\
\leq & F(t)+\frac{1}{2}(e(q-1))^{-2}|\Omega|+\frac{1}{2}(e \mu)^{-2}|\Omega|+\frac{1}{2}(e \mu)^{-2} C^{p(q-1+\mu)} p^{q-1+\mu} F(t)^{q-1+\mu},
\end{aligned}
$$

where we used $\left|x^{q-1} \log x\right| \leq(e(q-1))^{-1}$ for $0<x<1$ while $x^{-\mu} \log x \leq(e \mu)^{-1}$ for $x \geq 1, \mu>0$, and $C$ is the Sobolev constant satisfying $\|u\|_{p(q-1+\mu)} \leq C\|\nabla u\|_{p}$. By (4.7) and 4.8, we obtain

$$
F^{\prime}(t) \leq F(t)+\frac{1}{2}(e(q-1))^{-2}|\Omega|+\frac{1}{2}(e \mu)^{-2}|\Omega|+\frac{1}{2}(e \mu)^{-2} C^{p(q-1+\mu)} p^{q-1+\mu} F(t)^{q-1+\mu} .
$$

Integrating the above inequality over $(0, t)$, we see that

$$
\int_{F(0)}^{F(t)} \frac{d y}{y+\frac{1}{2}(e(q-1))^{-2}|\Omega|+\frac{1}{2}(e \mu)^{-2}|\Omega|+\frac{1}{2}(e \mu)^{-2} C^{p(q-1+\mu)} p^{q-1+\mu} y^{q-1+\mu}} \leq t .
$$

Applying Theorem 2.5, we get $\lim _{t \rightarrow T^{*-}} F(t)=+\infty$. Hence, we obtain a lower bound for $T^{*}$ estimated by

$$
T^{*} \geq \int_{F(0)}^{\infty} \frac{d y}{y+\frac{1}{2}(e(q-1))^{-2}|\Omega|+\frac{1}{2}(e \mu)^{-2}|\Omega|+\frac{1}{2}(e \mu)^{-2} C^{p(q-1+\mu)} p^{q-1+\mu} y^{q-1+\mu}} .
$$

The proof is complete. Here, it needs to noted that $C$ in the text is constant, and the $C$ in each row and even in the same row is different.

\section{Acknowledgments}

We are very grateful to the anonymous referees for their valuable suggestions that improved the article. The work is supported by the National Natural Science Foundation of China (No. 12171054) and the "Thirteen Five" Scientific and Technological Research Planning Project of the Department of Education of Jilin Province in China (JJKH20190547KJ, JJKH20200727KJ). 


\section{References}

[1] J. L. R. D'Alembert, Recherches sur la courbe que forme une corde tendue mise en vibration, Hist. Mm. Acad. Roy. Sci. Toulouse. 1747 (1750), 214-219.

[2] H. Ding and J. Zhou, Global existence and blow-up for a mixed pseudo-parabolic p-Laplacian type equation with logarithmic nonlinearity, J. Math. Anal. Appl. 478 (2019), no. 2, 393-420.

[3] P. Drábek and S. I. Pohozaev, Positive solutions for the p-Laplacian: Application of the fibering method, Proc. Roy. Soc. Edinburgh Sect. A 127 (1997), no. 4, 703-726.

[4] F. Gazzola and M. Squassina, Global solutions and finite time blow up for damped semilinear wave equations, Ann. Inst. H. Poincaré Anal. Non Linéaire 23 (2006), no. 2, 185-207.

[5] B. Guo and F. Liu, A lower bound for the blow-up time to a viscoelastic hyperbolic equation with nonlinear sources, Appl. Math. Lett. 60 (2016), 115-119.

[6] Y. He, H. Gao and H. Wang, Blow-up and decay for a class of pseudo-parabolic pLaplacian equation with logarithmic nonlinearity, Comput. Math. Appl. 75 (2018), no. 2, 459-469.

[7] C. N. Le and X. T. Le, Global solution and blow-up for a class of p-Laplacian evolution equations with logarithmic nonlinearity, Acta Appl. Math. 151 (2017), 149-169.

[8] H. A. Levine, Some nonexistence and instability theorems for solutions of formally parabolic equations of the form $P u_{t}=-A u+\mathscr{F}(u)$, Arch. Rational Mech. Anal. 51 (1973), 371-386.

[9] W. Lian, M. S. Ahmed and R. Xu, Global existence and blow up of solution for semilinear hyperbolic equation with logarithmic nonlinearity, Nonlinear Anal. 184 (2019), 239-257.

[10] _ Global existence and blow up of solution for semi-linear hyperbolic equation with the product of logarithmic and power-type nonlinearity, Opuscula Math. 40 (2020), no. 1, 111-130.

[11] W. Lian, J. Wang and R. Xu, Global existence and blow up of solutions for pseudoparabolic equation with singular potential, J. Differential Equations 269 (2020), no. 6, 4914-4959. 
[12] W. Lian and R. Xu, Global well-posedness of nonlinear wave equation with weak and strong damping terms and logarithmic source term, Adv. Nonlinear Anal. 9 (2020), no. 1, 613-632.

[13] J.-L. Lions, Quelque méthodes de résolution des problèmes aux limites non linéaires, Dunod, Paris, 1969.

[14] L. Ma and Z. B. Fang, Energy decay estimates and infinite blow-up phenomena for a strongly damped semilinear wave equation with logarithmic nonlinear source, Math. Methods Appl. Sci. 41 (2018), no. 7, 2639-2653.

[15] L. C. Nhan and L. X. Truong, Global solution and blow-up for a class of pseudo p-Laplacian evolution equations with logarithmic nonlinearity, Comput. Math. Appl. 73 (2017), no. 9, 2076-2091.

[16] M. Ohta, Remarks on blowup of solutions for nonlinear evolution equations of second order, Adv. Math. Sci. Appl. 8 (1998), no. 2, 901-910.

[17] K. Ono, On global existence, asymptotic stability and blowing up of solutions for some degenerate non-linear wave equations of Kirchhoff type with a strong dissipation, Math. Methods Appl. Sci. 20 (1997), no. 2, 151-177.

[18] L. E. Payne and D. H. Sattinger, Saddle points and instability of nonlinear hyperbolic equations, Israel J. Math. 22 (1975), no. 3-4, 273-303.

[19] M. Del Pino and J. Dolbeault, Nonlinear diffusions and optimal constants in Sobolev type inequalities: Asymptotic behaviour of equations involving the p-Laplacian, C. R. Math. Acad. Sci. Paris 334 (2002), no. 5, 365-370.

[20] M. Del Pino, J. Dolbeault and I. Gentil, Nonlinear diffusions, hypercontractivity and the optimal $L^{p}$-Euclidean logarithmic Sobolev inequality, J. Math. Anal. Appl. 293 (2004), no. 2, 375-388.

[21] D. H. Sattinger, On global solution of nonlinear hyperbolic equations, Arch. Rational Mech. Anal. 30 (1968), 148-172.

[22] J. Simon, Compact sets in the space $L^{p}(0, T ; B)$, Ann. Mat. Pura Appl. (4) 146 (1987), 65-96.

[23] X. Wang and R. Xu, Global existence and finite time blowup for a nonlocal semilinear pseudo-parabolic equation, Adv. Nonlinear Anal. 10 (2021), no. 1, 261-288.

[24] G. F. Webb, Existence and asymptotic behavior for a strongly damped nonlinear wave equation, Canadian J. Math. 32 (1980), no. 3, 631-643. 
[25] R. Xu, W. Lian, X. Kong and Y. Yang, Fourth order wave equation with nonlinear strain and logarithmic nonlinearity, Appl. Numer. Math. 141 (2019), 185-205.

[26] R. Xu and J. Su, Global existence and finite time blow-up for a class of semilinear pseudo-parabolic equations, J. Funct. Anal. 264 (2013), no. 12, 2732-2763.

Ying Chu, Yuqi Wu and Libo Cheng

School of Mathematics and Statistics, Changchun University of Science and Technology, Changchun, China

E-mail addresses: chuying_12345@sina.com, wuyuqi0903@163.com, clbyy@126.com 